\title{
Encuadres de la violencia machista: estudio comparativo de las noticias sobre delitos con víctimas (1996-2016)
}

\section{Frames of sexist violence: a comparative study with news reports about crime with victims (1996-2016)}

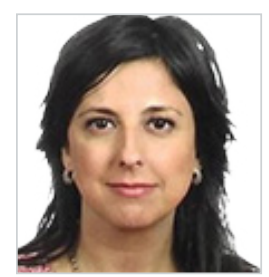

\begin{abstract}
María Gorosarri González. Profesora en UPV/EHU. Licenciada en Derecho (2002) y en Periodismo (2004). Doctora Internacional en Comunicación (2011), con la tesis (en euskara y en inglés) "Albisteen kalitatea: Research on Basque Media's News Quality", financiada por el Gobierno Vasco (2006-2010), así como varias de las estancias en Humboldt Universität (Berlín, Alemania) en 2009 y 2010. Ha realizado dos proyectos posdoctorales: sobre la calidad de la prensa europea de referencia (2000-2010), financiada por UPV/EHU (2012-2013), y un estudio de observación no participante en la redacción de varias televisiones alemanas, como investigadora en Freie Universität (Berlín, Alemania), financiada por el Gobierno Vasco (2014-2016). Sus líneas de investigación son la perspectiva feminista en el periodismo, calidad de las noticias y medios en lenguas europeas minoritarias.

Universidad del País Vasco, España

maria.gorosarri@ehu.eus

ORCID: 0000-0003-3890-8412
\end{abstract}

Recibido: 05/10/2020 Aceptado: 27/04/2021 - En edición: 25/05/2021 - Publicado: 14/06/2021 Resumen:

Este artículo analiza el tratamiento de los delitos con víctimas en los dos periódicos de referencia en España, El País y El Mundo. Siguiendo la teoría del enfoque ('framing'), mediante el análisis de contenido, se medirán cuatro encuadres habituales que inciden en la consideración social de la violencia machista: identificación más detallada de la denunciante que del agresor, construcción de la alteridad racial del agresor, atribución explícita de responsabilidad en el delito sufrido y menoscabo de la credibilidad de la denunciante, condicionado a la reiteración del término 'presunto' (Berganza, 2003; Carbadillo, 2010; Easteal, Holland y Judd, 2015; Escribano, 2014). Se comparará por primera vez el alcance de esos cuatro encuadres en violencias machistas con otros delitos que causan víctimas y necesitan de la denuncia de éstas (terrorismo, delitos de odio, siniestralidad laboral, seguridad vial, delitos contra la propiedad y violencia contra las personas sin componente
Received: 05/10/2020 - Accepted: 27/04/2021 - Earlyaccess: 25/05/2021 - Published: 14/06/2021 Abstract:

This article analyses the treatment of news about crime in the two reference newspapers in Spain, El País y El Mundo. Assessing the theory of framing, following content analysis research, four usual frames will be measured, due to the high impact on the social consideration of violence against women they have: more detailed identification of the accuser than the perpetrator, the construction of racial otherness about the perpetrator, explicit attribution of responsibility in the undergone crime, and the reduction of accuser's credibility, determined by the repetition of the expression 'alleged crime' (Berganza, 2003; Carbadillo, 2010; Easteal, Holland \& Judd, 2015, Escribano, 2014). The significance of those four frames in the news about violence against women will be compared for the first time with other felonies that cause victims and need the accusation of those (terrorism, hate crimes, work accidents, road security, crimes against property,

Cómo citar este artículo:

Gorosarri González, M. (2021). Encuadres de la violencia machista: estudio comparativo de las noticias sobre delitos con víctimas (1996-2016). Doxa Comunicación, 32, pp. 75-94.

https://doi.org/10.31921/doxacom.n32a4 
de género). Se probará que la identificación más detallada de la denunciante, la alteridad racial del agresor y el menoscabo de la credibilidad de la denunciante son encuadres específicos a las violencias contra las mujeres. El estudio temporal (1996-2016) evidenciará, además, que se acentúan tras la aprobación de la legislación sobre igualdad.

Palabras clave:

Violencia contra las mujeres; violencia de género; presunto; framing; delitos. and non-gendered violence against people). It will be proved that the more detailed identification of the accuser, the construction of racial otherness about the perpetrator and the reduction of accuser's credibility are specific frames for violence against women. Finally, the temporal analysis (1996-2006) will evidence that they got emphasized after the passing of Equal Rights Acts.

\section{Keywords:}

Violence against women; gender-based violence; alleged; framing; crime.

\section{Introducción, estado de la cuestión y objetivo de la investigación}

Los medios de comunicación centran su función social en explicar la realidad, dirigiendo su atención hacia aquellos acontecimientos que consideran relevantes. Describen esa realidad a través de narrativas que organizan su discurso, denominadas 'frames' o marcos. Estos "esquemas de interpretación" (McCombs, 2006: 87-99) o "guías de conocimiento con respecto a un tema o problema determinado" (Carbadillo, 2010: 221-224) orientan la percepción de la audiencia sobre lo acontecido. La propia actividad periodística constituye un proceso de elaboración de 'frames' o marcos, ya que analizar la realidad implicar la "creación de significados" (Escribano, 2014: 19). Todo ello incide en la consideración social hacia tales cuestiones, especialmente en el largo plazo.

Ardèvol-Abreu diferencia el encuadre de la intencionalidad en la selección de las noticias ('agenda setting'), señalando que este último determina qué es noticia, mientras que el encuadre se centra en "la manera en que se describe el tema o acontecimiento, así como el esquema interpretativo que ha sido activado para procesarlo" (2015: 427). De esa manera, los medios y su público comparten "un contexto común de significaciones simbólicas" y es, a partir de estos esquemas transmitidos a través de las noticias, cómo interpretan esa realidad social (Ardèvol-Abreu, 2015: 429; Giménez y Berganza, 2009: 61).

La creación de los 'frames' o encuadres es un proceso en el que los medios eligen ciertos atributos de las informaciones sobre determinadas noticias. A este proceso se le denomina 'frame building' y, como consecuencia, transmite la manera de evaluar el tema que otorga el medio a esa cuestión (Ardèvol-Abreu, 2015: 425-426). Mediante el estudio de esos encuadres, se pretende "captar los efectos latentes" que ejercen los medios en la definición de la realidad social (Carbadillo, 2010: 217-220). Por ello, los medios y su expresión periodística dan forma a la consideración de la violencia machista que se comparte en sociedad.

\subsection{Encuadres habituales en las noticias sobre violencias machistas}

La investigación académica sobre los encuadres mediáticos ha mostrado dos vías de estudio. La vía inductiva consiste en analizar una parte del total de la muestra e identificar algunos 'frames' sobre el objeto de estudio. La vía deductiva, por 
el contrario, se ha decantado por seleccionar, en primer término, los encuadres a analizar y su estudio ha consistido en determinar el alcance de éstos (Ardèvol-Abreu, 2015: 434-435).

La investigación de las noticias sobre violencias machistas ha identificado diversos encuadres que inciden en la falta de consideración de "problema social" del fenómeno, tales como, el tratamiento episódico del crimen y la consideración de suceso del mismo (Berganza, 2003: 9-10). Otros cuatro encuadres habituales se centran en las figuras de autor y víctima: la identificación más detallada de la denunciante que del agresor, la construcción de la alteridad racial del autor, la atribución explícita de responsabilidad en el delito sufrido, y el menoscabo de la credibilidad de la denunciante (Easteal, Holland y Judd, 2015: 111).

En primer lugar, los medios explican quién es el sujeto de la noticia. La diferente caracterización de autor y víctima ha expuesto la cuestión de la innecesaria identificación de la persona agredida, para no atraer la atención hacia ella, sino hacia los agresores (Escribano, 2014: 73-78). Igualmente, se ha destacado el hecho de que las mujeres aparecen identificadas por su relación familiar (De Frutos, 2015).

No obstante, la ley no impide ofrecer los datos identificativos y fotografías del detenido, siempre y cuando se respete la presunción de inocencia hacia el mismo. Sin embargo, son los propios medios quienes se autocensuran y prefieren emplear sus iniciales, especialmente en los delitos de mayor estigma social (El País, 2014: 28).

En segundo lugar, el 'frame' sobre la construcción de la alteridad racial del autor tiene como base la creencia de que la violencia contra las mujeres se trata de un problema migrado. En efecto, tal alteridad se ha traducido en la imagen de hombres migrantes como cierto tipo de criminales violentos: "violadores, pedófilos y asesinos de personas inocentes y respetuosas con la ley" (De Noronha, 2018: 353). Por ello, se recomienda no especificar el origen del agresor, cuando no sea relevante (El Mundo, 2002: 68). Este encuadre resulta medible atendiendo a los datos sobre la nacionalidad, procedencia y/o religión de denunciante y denunciado. La academia ha demostrado precisamente que los delitos que incluyen tales datos aparecen desproporcionalmente con mayor frecuencia en los medios (Carbadillo, 2010: 309-316; Sutherland et al, 2016).

En tercer lugar, la atribución de la responsabilidad del delito a la víctima o al agresor permite identificar el fenómeno conocido con el término 'revictimización' ('victim blaming', en inglés), es decir, la atribución explícita o implícita de la responsabilidad del delito a la propia víctima, por ejemplo, señalando la cuestión de si había cursado denuncia por violencia de género anteriormente a ser asesinada por un hombre (Peris Vidal, 2016: 1131-1132). Indirectamente, justificar, anular o mitigar la responsabilidad del agresor por cuestiones emocionales o situaciones de enajenación por estupefacientes también repercute en culpabilización hacia la víctima, al igual que la presentación del agresor de manera deshumanizada, como un enfermo (Escribano, 2014: 73-78; Tiscareño y Miranda, 2020: 54-56). Por todo ello, se estima oportuna la inclusión en la información de los antecedentes judiciales del agresor o, como exige RTVE, de las medidas de protección adjudicadas, ya que estas dos últimas cuestiones apuntarían a la responsabilidad fallida del propio Estado en la salvaguarda de la vida de sus ciudadanas (Sutherland et al, 2016).

Por último, el cuarto encuadre alude al menoscabo de la credibilidad de la denunciante. En el caso de las violencias contra las mujeres, la persecución de tales delitos exige la denuncia previa, normalmente, de la mujer agredida, que será 
quien inicie el proceso. En muchos de los casos denunciados, además, el testimonio de la mujer será la principal prueba inculpatoria contra el acusado. Por ello, el descrédito del relato de la víctima se lleva a cabo mediante el empleo de determinadas expresiones, como el término "presunto" (Easteal et al., 2015: 109). Si bien no se ha estudiado la extensión de su empleo, tangencialmente se ha detectado una presencia excesiva del mismo: siete veces en una noticia sobre acoso sexual "de trescientas palabras" (Judd y Easteal, 2013: 17).

La presente investigación pretende superar un vacío de la investigación: el estudio comparativo de los cuatro encuadres en las noticias sobre violencias contra las mujeres y el resto de delitos del Código Penal español que exigen la denuncia de la víctima. Así, se analizará también el alcance del cuarto encuadre aún no estudiado hasta el momento: el que atiende al grado de credibilidad que se otorga en la noticia al testimonio de la persona agredida. Siendo la presunción de inocencia un derecho fundamental reconocido en el artículo 24.2 de la Constitución Española, "se trata de un principio general orientador de la mente judicial para conseguir preservar la imparcialidad del juzgador" (Nieva, 2016). La academia ha criticado el excesivo empleo del término 'presunto', subrayando que no existe la presunción de culpabilidad, sino la de inocencia. En ese sentido, RTVE recomienda el uso del término 'sospechoso':

"La presunción de inocencia supone que toda persona es inocente hasta que exista una sentencia judicial que declare su culpabilidad. Si se comete un asesinato y la policía detiene a una persona, al decir "presunto asesino» estamos diciendo que esa persona es culpable salvo que se demuestre su inocencia. Además, esa forma de hablar hace que la opinión pública condene socialmente al detenido antes de ser juzgado. Por ello, se sugiere que se utilice «sospechoso»" (RTVE, 2008).

Priorizar el término ‘supuesto' en lugar de 'presunto' es una vieja cuestión en los medios españoles. Ya en 1998, Francisco Gor, como defensor del lector en El País, expuso:

"El término "presunto" ha llegado a adquirir en el lenguaje periodístico carácter sustantivo para designar a quien, no habiendo sido condenado por los jueces, se le considera poco menos que en capilla a la espera de una sentencia inexorable, por supuesto condenatoria. (...) Desde el punto de vista jurídico, no hay duda de la improcedencia de aplicar el término "presunto» a otra realidad o circunstancia que no sea la inocencia del implicado en un proceso. (...) Al aplicar presunto al autor de un delito estamos diciendo lo contrario de lo que pretendemos: no hay presuntos homicidas, sino aparentes homicidas, presuntos inocentes hasta la sentencia firme. El éxito periodístico de la expresión, traída sin más de la expresión constitucional presunción de inocencia, la ha incorporado a nuestro lenguaje (...)” (Gor, 1998).

De hecho, el incorrecto empleo del término 'presunto' en el contexto mediático como sinónimo de 'supuesto' acarreó su inclusión en el diccionario de la Real Academia Española (RAE) a partir de $22^{\text {a }}$ edición, en 2001, en referencia al acusado que no ha sido condenado aún. Sin embargo, tal referencia se eliminó en 2014 (Fundeú, 2016). Por eso mismo, tradicionalmente se ha considerado que 'presunto' no es siempre sinónimo de 'supuesto':

“«Presunto» designa a quien se considera posible autor de un delito, cuando se han abierto diligencias procesales pero aún no hay fallo de la sentencia, y se emplea «supuesto» cuando existen indicios de criminalidad pero no se ha abierto causa judicial" (Fundéu, 2011).

En la misma línea, RTVE recomienda el empleo de 'presunto' para quien ya está siendo investigado: 
"Presunto y supuesto: una persona es un «supuesto» delincuente cuando existen indicios de criminalidad pero no se le ha abierto causa judicial. En cambio, se debe emplear «presunto» cuando, por haber presunción de delito, se han abierto diligencias procesales, pero no hay fallo de la sentencia aún" (RTVE, 2008).

Sin embargo, no se habla de 'posible' como sinónimo ('posible homicida'). Siguiendo las recomendaciones del Defensor del Lector en El País, los medios se podrían referir al acusado ('encausado', desde la Reforma de la Ley de Enjuiciamiento Criminal de 2015) según el momento procesal en el que se encuentre (Gor, 1998). De la misma manera, El País (2014: 444) y RTVE (2018) apelan a emplear las figuras procesales correspondientes. Los estudios jurídicos también señalan la conveniencia de nombrar la autoría según el momento procesal:

"La tozuda realidad parece insistir en demostrar, por tanto, que entre el código binario reconocido por la Constitución, de «inocente» 0 «culpable» -tras la sentencia firme-, parecería existir una tercera vía que recorrería un largo camino desde el «sospechoso» hasta el «acusado», pasando por el «imputado» o «procesado», por no hablar de híbridos como «querellado» 0 «denunciado» (...)” (Sánchez-Vera, 2012: 35).

A pesar de ello, la costumbre periodística se ha impuesto y recientemente 'presunto' ha pasado a considerarse ya sinónimo de 'supuesto' (Fundéu, 2016). A su vez, la extensión del uso del término ha traído con ella una nueva manera incorrecta de utilizarlo en los medios, empleándolo incluso en ocasiones en las que todavía no hay un sujeto identificado y, por lo tanto, difícilmente imputable: "La policía busca al presunto [sic] asesino de la mujer y el menor de Alcobendas" ( $A B C, 02 / 05 / 2017)$. O, incluso, cuando el autor ya ha sido condenado: "La Audiencia de Bizkaia ha condenado a once años de prisión al exprofesor del colegio Gaztelueta acusado de presuntos [sic] abusos sexuales" (Eldiario.es, 15/11/2018). En consecuencia, la presunción de inveracidad en las denuncias de mujeres muestra la merma en la credibilidad hacia las mismas. El término 'presunto' se aplica como una duda sobre la propia denunciante: "La presunta [sic] víctima de la violación grupal en los sanfermines de 2016 declaró ayer en el juicio” (El Correo, 15/11/2017). Por ello, esta mala praxis ha tomado el nombre de 'presuntitis' (Gorosarri, 2016).

De hecho, según la jurisprudencia del Tribunal Supremo (TS), "a los medios se les impone la obligación a emitir una información veraz". Para ello, el TS diferencia veracidad de verdad, "ya que esta última requiere la verificación de las pruebas, carga más allá de la actividad periodística" (CGPJ, 2017). La investigación periodística únicamente permite relatar actos denunciados o señalar comportamientos injustos, sin poder definirlos como delitos. Por eso mismo, la información veraz será informar sobre la denuncia, sin poner en duda la veracidad de lo denunciado -duda implícita en la expresión 'presunta violación'-, a no ser que el periodista tenga pruebas sobre la falsedad de la denuncia o de los hechos denunciados. En efecto, el derecho a la información veraz se impone incluso sobre el derecho individual al olvido. El Tribunal Supremo ha rechazado la demanda de derecho al olvido de un hombre absuelto de doble asesinato, ya que la información del juicio celebrado, así como la foto publicada durante la vista del mismo, son "veraces" y obtenidas "con ocasión de un hecho noticiable" (CGPJ, 2017).

En consecuencia, el grado de credibilidad que los medios otorgan a las denunciantes de violencia machista resulta medible siguiendo la frecuencia del empleo de la expresión 'presunto autor', 'presunto delito' o, incluso, 'presunta víctima', 
comparándolos con el tratamiento mediático a los diferentes tipos de delitos a juzgar partiendo de la denuncia de la víctima.

\subsection{Objetivo y preguntas de investigación}

En resumen, son cuatro los 'frames' en las noticias que dan forma a la consideración social de autor y víctima de violencias contra las mujeres: la identificación más detallada de la denunciante que del agresor, la alteridad racial del agresor, la atribución explícita de responsabilidad y el menoscabo en la credibilidad de la denunciante. La presente investigación medirá por primera vez la magnitud de este cuarto encuadre, según la frecuencia del empleo de la palabra 'presunto'.

Así mismo, para considerar que estos cuatro 'frames' son específicos de la información sobre violencias contra las mujeres se debe comparar su alcance en el resto de noticias sobre delitos. Tal estudio comparativo implica analizar los delitos instruidos a partir de la denuncia de la parte agredida. En consecuencia, el presente artículo analizará el tratamiento informativo de otros delitos contra las personas y la propiedad, según el Derecho Penal español, también basados en la denuncia de la persona agredida. De esa manera, podremos identificar si los medios de comunicación españoles informan con la misma profesionalidad sobre la violencia contra las mujeres y sobre aquélla sin especificidad de género.

En consecuencia, el objetivo que ha guiado esta investigación es el siguiente: investigar si hay 'frames' específicos para las noticias sobre violencias machistas que difieren del tratamiento mediático de otros delitos con víctimas. Basándose en estudios anteriores, esta investigación pretende medir las diferencias en la identificación más detallada de la denunciante que del agresor, la construcción de la alteridad racial del agresor, la atribución explícita de responsabilidad en el delito sufrido y el menoscabo de credibilidad de la denunciante, de manera comparativa entre noticias sobre violencias contra las mujeres y el resto de informaciones sobre delitos con víctimas (Berganza, 2003; Carbadillo, 2010; Easteal et al., 2015: 111; Escribano, 2014).

En aras de responder al objeto de estudio, se han formulado las siguientes preguntas de investigación o 'research questions' (RQ):

RQ1: ¿Ofrecen los medios más datos identificativos de las víctimas femeninas que de las víctimas masculinas?

RQ2: ¿Identifican las noticias con mayor número de datos a los agresores extranjeros?

RQ3: ¿Desapareció la justificación al agresor con la aprobación de la legislación sobre igualdad?

RQ4: ¿Desacreditan los medios la credibilidad al testimonio de la víctima debido al empleo incorrecto del término 'presunto'?

RQ5: ¿Produjo la adopción de leyes en materia de igualdad algún cambio en la manera de informar sobre violencias machistas? 


\section{Metodología}

Los cuatro 'frames' propuestos serán estudiados siguiendo el método del análisis de contenido, técnica de investigación que hace replicable y válida la clasificación y evaluación del "contenido manifiesto y latente" de un material informativo (Krippendorf, 2018: 24). Investigaciones anteriores relativas al 'framing' de las noticias sobre violencias contra las mujeres también han empleado esta técnica de investigación (McManus y Dorfman, 2005; Marín, Armentia y Caminos, 2011).

Las noticias de los ejemplares que forman la muestra aleatoria, basada en la técnica de la semana compuesta, se han clasificado según las categorías de los delitos contra las personas y la propiedad: violencia de género (VG), violencia contra las mujeres fuera del ámbito de la pareja, agresiones sexuales, terrorismo, siniestralidad laboral, seguridad vial, delitos contra la propiedad y otros. En primer lugar, la categoría de violencia de género determina el ámbito de aplicación de la LOVG (2004), es decir, la violencia ejercida contra una mujer por aquel hombre con el que tiene o ha tenido una relación sentimental. En segundo lugar, la violencia contra las mujeres fuera del ámbito de la pareja alude a la violencia machista, cuando no ha existido relación amorosa alguna entre autor y víctima. De esa manera, se recogerán los casos de violencia contra las mujeres que no quedan amparados en la LOVG de 2004, al carecer del componente de afectividad entre víctima y victimario. En tercer lugar, las agresiones sexuales se han analizado como categoría independiente. Estas tres categorías juntas constituyen las denominadas violencias machistas o violencias contra las mujeres, aludiendo a la pluralidad y diversidad de estas violencias (Zurbano Berenguer, 2018: 84-85). En cuarto lugar, el terrorismo incluye las noticias sobre organizaciones terroristas. En quinto lugar, los delitos de odio se refieren a agresiones a personas del colectivo LGTBI o de otras razas. En sexto lugar, la siniestralidad laboral recoge los accidentes laborales. En séptimo lugar, la seguridad vial categoriza los accidentes de tráfico en los que se vean envueltas dos personas, al resultar esencial para el presente estudio las figuras de víctima y victimario. En ese sentido, en octavo lugar, los delitos contra la propiedad sólo serán analizados cuando la víctima del delito sea una persona física que deba cursar denuncia y no, el conjunto de la ciudadanía, excluyendo de esa manera los delitos de corrupción política y económica. Finalmente, las noticias sobre delitos que pudieran presentar ambas figuras (víctima y victimario) y que no se encuadren en las categorías anteriores serán catalogados bajo la etiqueta de 'otros'.

Para la recogida de datos, se ha elaborado una ficha de análisis (Tabla núm. 1) en la que se especificará a qué tipo de delito con víctimas se refiere cada noticia. Así mismo, se recogerán datos relativos al periodista que firma la información. A continuación, se analizarán las informaciones en relación a los cuatro encuadres elegidos. Por cuestiones prácticas, la diferente caracterización de autor y víctima (que denota quién es el sujeto que el medio considera de interés noticiable) señalará también si la identificación del agresor ha consistido en especificar su alteridad racial (Carbadillo, 2010: 309-316; Escribano, 2014: 73-78; Sutherland et al, 2016).

Seguidamente, se recogerán las cuestiones de atribución de responsabilidad, si las hubiere. Se entenderá que la noticia atribuye explícitamente la responsabilidad a la víctima, cuando se mencione la cuestión sobre la existencia o no de denuncia previa. La información sobre antecedentes del agresor recalcará la intencionalidad de éste, a la vez que denota la negligencia del Estado en prevenir el crimen. Igualmente, el asunto sobre las medidas de protección señala beligerancia social de los medios para con las medidas legales de protección a los derechos de las mujeres. Finalmente, la justificación 
de lo sucedido, aludiendo al alcohol, celos, enfermedad, etc... dará a entender el carácter episódico de la violencia y no, como recoge la LOGV (2004), "el símbolo más brutal de la desigualdad existente en nuestra sociedad" (Peris Vidal, 2016: 1131-1132).

En último lugar, el grado de credibilidad otorgado por el medio a la denunciante se medirá contabilizando los términos 'presunto’ y ‘supuesto' en la noticia (Easteal et al., 2015: 109). Se especificarán si se refieren al agresor (empleo correcto), a la víctima (mala praxis) o al delito (empleo incorrecto).

Tabla 1. Ficha de análisis creada

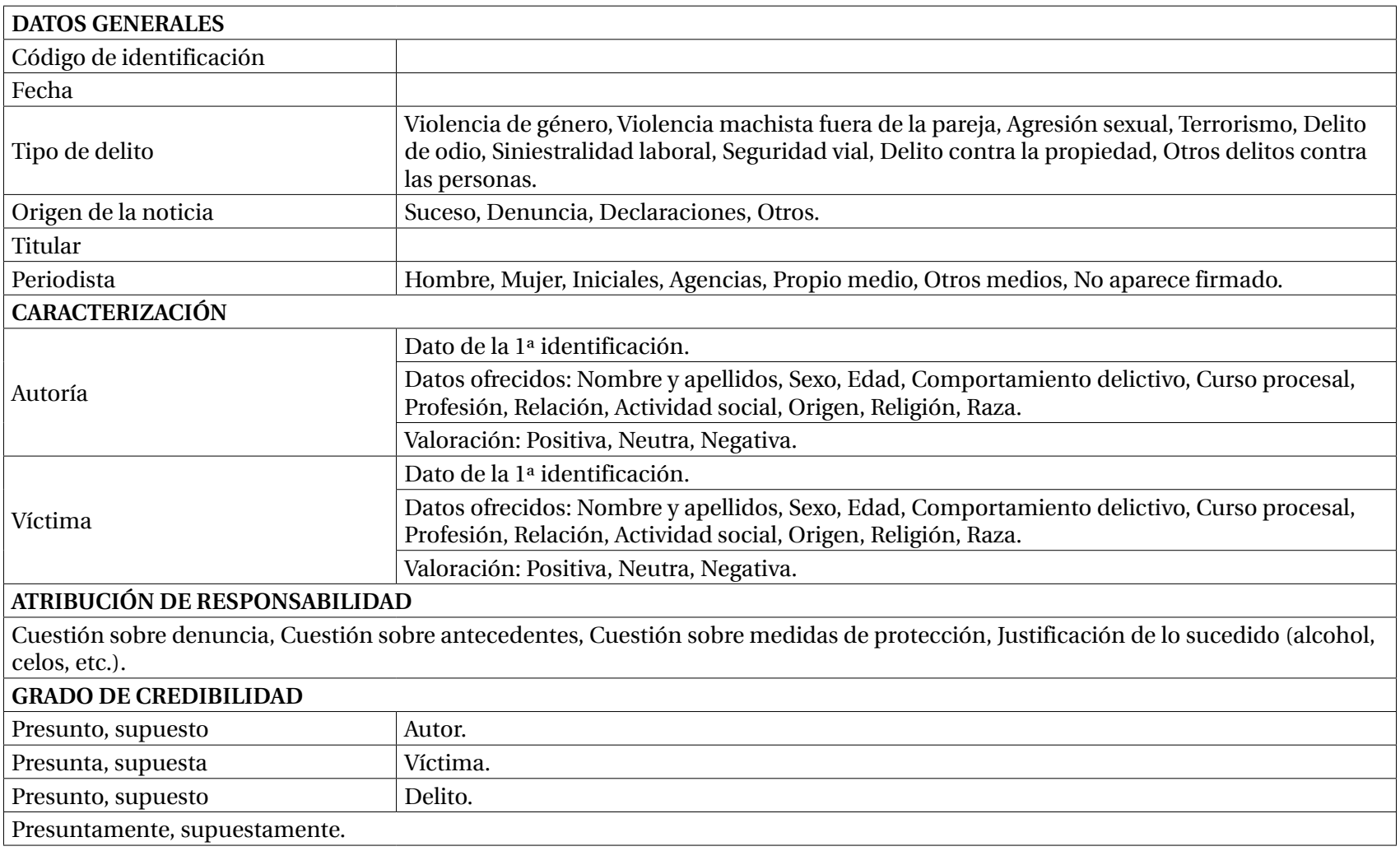

\section{Fuente: elaboración propia}

Para asegurar que la codificación se ajusta a las normas académicas, se ha comprobado la fiabilidad de la ficha de análisis desarrollada. A pesar de tratarse de una única investigadora, la fiabilidad intracodificadora debe quedar probada. Así, después de analizar 38 noticias (10,19\% de la muestra), dos veces, con un intervalo de quince días entre cada codificación, la fórmula de Holsti que mide el grado de coincidencia en las decisiones codificadoras, también denominado acuerdo 
simple, otorga un $99,2 \%$ de fiabilidad. Igualmente, dos codificadores externos analizaron 20 noticias iguales (5,36\% de la muestra). La fiabilidad intercodificadora fue, así, del 98,65\%, superior al 90\% requerido (Lacy et al., 2015: 792-796).

El universo de análisis de la presente investigación está compuesto por los dos periódicos generalistas españoles de mayor tirada (El País y El Mundo) en los últimos veinte años, es decir, de 1996 a 2016. Dicho período resulta idóneo para analizar las tendencias informativas en relación a las noticias sobre violencia contra las mujeres. De un lado, la muestra comienza en los años de implantación del nuevo Código Penal de 1995. De otro, los cambios legislativos en el mismo en materia de igualdad en diciembre de 2004 (Ley Orgánica de Medidas de Protección Integral contra la Violencia de Género -en adelante, LOVG-) y en marzo de 2007 (Ley Orgánica Para la Igualdad Efectiva de Mujeres y Hombres) quedan recogidos en la muestra, ya que la mitad de la misma sería anterior a dichas leyes y la otra mitad, posterior.

En total, la muestra aleatoria queda compuesta de 42 números (siete números de cada año a analizar) de cada medio, es decir, 84 ejemplares (dos medios). En cada ejemplar, se han seleccionado las noticias referentes a delitos con víctimas recogido en el Código Penal español. Así, la muestra de la presente investigación ha quedado compuesta por 373 noticias: 205 recogidas en El País y 168, en El Mundo. De esa manera, la unidad de análisis de la investigación será la noticia. El total de noticias seleccionadas han sido estudiadas siguiendo el método de análisis de contenido. Para la medición empírica de los encuadres mediáticos se ha seguido el análisis deductivo, es decir, se han seleccionado a priori los cuatro encuadres a estudiar.

Trabajos anteriores han demostrado que la semana compuesta es la muestra más eficiente en cuanto a costes y la más objetiva para tratar los temas a analizar en los diarios (Lacy et al., 2001). Además, para observar las tendencias a lo largo de un período, dicha muestra debe mantener un intervalo constante (Luke et al., 2011). En nuestro caso, el intervalo de la muestra será de cuatro años. De esa manera, analizaremos una semana compuesta cada cuatro años del período incluido en la muestra, que se corresponderá con los siguientes años: 1996, 2000, 2004, 2008, 2012 y 2016.

Finalmente, las investigaciones sobre el número necesario de unidades de muestra han demostrado que seis semanas compuestas ofrecen datos precisos, sea cual sea el tamaño del universo a analizar (Luke et al., 2011). Por lo tanto, la presente investigación analizará un total de seis semanas compuestas de cada periódico en los años arriba indicados.

\section{Resultados}

Las noticias sobre delitos ejercidos por una persona con consecuencias sobre otra persona o sus bienes constituyen el 4,48\%de las noticias publicadas en El País y el 3,67\%, en El Mundo, en el período analizado. Entre todas ellas, las más numerosas son las relativas a atentados terroristas (1,46\% en El País y 1,04\% en El Mundo) y aquéllas sobre violencias contra las mujeres -violencia de género, violencia machista fuera del ámbito de la pareja y agresiones sexuales- (1,33\% en El País y $1 \%$ en El Mundo). Las violencias machistas no obtienen el liderazgo de importancia en número hasta 2016, al reducirse la información sobre terrorismo. En segundo lugar, destacan las noticias sobre otras violencias contra las personas no relativas a sexo ni ideología $(0,75 \%$ y $0,85 \%)$. Finalmente, los delitos contra la propiedad $(0,32 \%$ y $0,27 \%)$ son más frecuentes que los de seguridad vial $(0,27 \%$ y $0,21 \%)$ y los accidentes laborales $(0,26 \%$ y $0,21 \%)$. 
Teniendo en cuenta que el 13,86\% de las noticias sobre delitos con víctimas en El País y el 43,24\% en El Mundo no aparecen firmadas, aquéllas elaboradas por periodistas constituyen únicamente el 54,99\%, de promedio. Comprobamos que periodistas hombres firman más noticias que periodistas mujeres ( $26,1 \%$ y $16,78 \%$, respectivamente). Ellos se imponen en todas las noticias sobre delitos, excepto en seguridad vial, donde las firmas femeninas son más del doble de las masculinas. A partir de 2008, disminuyen las informaciones elaboradas por mujeres periodistas sobre violencia de género y violencia sexista fuera del ámbito de la pareja, a la vez que aumenta el número de noticias sobre agresiones sexuales y otras violencias contra las personas firmadas por hombres periodistas.

De esa manera, las noticias elaboradas por mujeres periodistas destacan en mayor proporción en la sección local, donde se publican los accidentes de tráfico, mientras que las noticias sobre violencias contra las mujeres (violencia de género, violencia machista fuera del ámbito de la pareja y agresiones sexuales), clasificadas en sociedad, presentan principalmente iniciativa informativa ajena la redacción $(35,835 \%)$ o aparecen firmadas por hombres $(31,28 \%)$ y, en menor medida, elaboradas por mujeres $(21,29 \%)$.

A continuación, para analizar la diferente caracterización de autor y víctima que presentan los medios, nos centraremos en los datos que ofrecen de los mismos. En primer lugar, casi un cuarto de las noticias sobre delitos cuyas víctimas no son únicamente mujeres evita la figura del autor en el titular. En el caso de las violencias contra las mujeres, sólo el 12,26\% obvia al autor. Así, el agresor es aludido con mayor frecuencia en los delitos de violencias machistas y el dato con el que inicialmente le identifican los medios es la relación entre agresor y víctima $(14,23 \%)$-amorosa, familiar, vecinal, amistad, laboral, comercial...-. En el resto de delitos, por el contrario, el primer dato del autor es la relevancia social de éste $(21,94 \%)$-actividad laboral, activismo social, etc.-; por ejemplo: "Detenido en Cádiz un futbolista por agredir a su esposa" (El País, 05/06/2008).

Según el tipo de delito, los medios tienden a presentar al autor en base a una característica diferente. Así, el comportamiento delictivo se utiliza para identificar por primera vez al autor de delitos contra la propiedad $(41,19 \%)$ y de terrorismo (25,33\%), por ejemplo: “Dos ladrones causan 20 heridos al chocar en una persecución” (El Mundo, 23/07/2004). La violencia machista fuera del ámbito de la pareja destaca por ser el delito que con mayor frecuencia identifica al autor según su identidad (12,94\%) y, por el contrario, el sexo resulta el elemento identificativo esencial en las noticias sobre violencia de género ("un hombre"), en un 31,77\% de las noticias. La identificación mediante el sexo del agresor en el resto de delitos es, de media, un 3,75\%. A pesar de que para la calificación del delito de violencia de género se requiere una relación afectiva entre agresor y víctima, resulta diez veces más común la primera identificación del agresor de violencia de género por su sexo que en el resto de delitos.

De la misma manera, el origen del autor únicamente resulta el primer elemento identificativo en las violencias contra las mujeres $(4,83 \%)$-especialmente, en las agresiones sexuales (11,54\%), aunque esa tendencia se haya frenado desde 2008-, frente al $0,74 \%$ del resto de delitos. Es decir, el autor es identificado en primer lugar según su origen cuatro veces más frecuentemente que en el resto de delitos; y en las agresiones sexuales, hasta diez veces más.

Ahora, atendiendo al total de detalles sobre la persona del autor que ofrecen los medios, las noticias sobre violencias contra las mujeres presentan mayor cantidad de datos sobre el agresor que el resto de delitos. En concreto, se revelan 
datos sobre la identidad del autor el doble de veces que en el resto de delitos, aunque desde 2008 se identifica con menor frecuencia al autor. Igualmente, la relación entre autor y víctima es revelada en el 16,63\% de las noticias sobre violencias contra las mujeres, incluyendo agresiones sexuales ("Denuncia contra un alcalde por violación: Una edil malagueña asegura que posee grabaciones de las vejaciones que sufrió", El País, 25/07/2008 y “Arrestado un matrimonio de La Palma por abusar sexualmente de su hija, con deficiencia psíquica”, El Mundo, 05/06/2008). En el resto de delitos, la relación entre autor y víctima se recoge únicamente en el 3,31\%, es decir, cinco veces menos. Al contrario, estos últimos priman la consideración social del autor (30,45\%), muy por encima que las noticias sobre las violencias contra las mujeres (11,36\%). Sin embargo, el origen del autor es mencionado con mayor frecuencia en los casos de violencias machistas (26,68\%), aunque también es un elemento importante en la descripción del autor del resto de delitos (16,57\%), si bien no es el primer dato identificativo del autor que dan. No obstante, a partir de 2008, las noticias sobre violencia machista fuera de la pareja y agresiones sexuales redujeron a la mitad los datos sobre el origen de los agresores, al mismo tiempo que las informaciones sobre violencia de género doblaban el número de relatos con esos datos.

Respecto a la presentación que los medios ofrecen de la víctima de delitos, sus datos identificativos aparecen con mayor frecuencia que los del agresor: sólo el 13,76\% de las noticias no identifican a la víctima y el 19,89\%, al autor. Sin embargo, las víctimas de violencia de género y de violencia machista fuera de la pareja son quienes con mayor relevancia aparecen identificadas ("La juez deja en libertad al ex novio de Sara Alonso y encarcela a su amigo", El País, 07/11/2004) y se refuerza a partir de 2008. De esa manera, la violencia machista fuera de la pareja $(28,83 \%)$ y terrorismo $(14,46 \%)$ son las que con mayor frecuencia identifican en primer lugar a la víctima por su identidad, respecto a un $13,13 \%$ de media en todos los delitos. Las violencias contra las mujeres destacan, otra vez, por presentar a la víctima en primer término según su relación con el agresor en el 30,97\% de las noticias (tendencia en alza a partir de 2008). Cabe destacar que únicamente en un tipo de violencia machista, la violencia de género, se requiere de una relación afectiva entre agresor y víctima. Al contrario, en el resto de delitos sólo en el 4,5\% de las noticias se alude a la relación de víctima y autor para presentar la noticia, ya que para éstos resulta más relevante la condición social de la víctima $(17,34 \%)$-"Suspendidos de servicio varios guardias civiles por la muerte de un porteador marroquí en Ceuta" (El Mundo, 14/04/2004)- y su origen (11,80\%) -"La policía colombiana desconoce quién secuestró al español Ángel Blanco" (El Mundo, 03/01/2000)-. Así, el número de noticias sobre violencia de género que identifica a la víctima en primer lugar según su relación con el autor es superior a aquéllas que presentan al agresor en relación a su vínculo con la víctima.

En cuanto al conjunto de datos ofrecidos por los medios para identificar a la víctima, éstos son más numerosos en las noticias sobre violencias contra las mujeres, destacando la edad (53,94\%, frente al 28,57\% en el resto de delitos) y la relación con el agresor (18,13\%, frente al 3,34\%), detalles que se ofrecen con mayor frecuencia a partir de 2008. Ya se ha señalado anteriormente que para los delitos de violencia machista fuera de la pareja y agresiones sexuales la afectividad no es un requisito legal. Así mismo, es de subrayar que las agresiones sexuales son las únicas noticias sobre violencias contra las mujeres que ofrecen tres veces mayor cantidad de datos del autor que de la víctima. Sin embargo, al identificar a la denunciante según la relación social con el agresor, resulta fácilmente reconocible.

Finalmente, el origen de la víctima aparece en el 19,96\% de las noticias sobre violencias machistas y en el 23,38\% del resto de delitos, quienes también incluyen datos sobre la raza $(3,14 \%)$ y la religión $(1,34 \%)$ de la víctima. De ese modo, compro- 
bamos que en las noticias sobre violencias contra las mujeres se incluye con mayor frecuencia el origen del autor que el de la víctima, especialmente en las informaciones sobre agresiones sexuales, aunque este dato desaparece de las noticias a partir de 2008. Por el contrario, en el resto de delitos se identifica a la víctima más recurrentemente según su origen que al autor.

En ese sentido, la primera identificación mediante datos relativos al nombre y apellidos es cuatro veces más frecuente para víctimas que agresores en las noticias sobre violencia de género, especialmente tras la aprobación de la legislación que combate ésta. Se mantiene esa misma proporción en la primera presentación de la víctima, identificándola por su relación con el agresor (“Detenido por agredir a su exnovia en Bilbao", El País, 11/04/2012).

Así mismo, atendiendo a la valoración que la redacción de la noticia hace del autor, éste es presentado de manera neutral en los delitos cuyas denunciantes no son únicamente mujeres con mayor frecuencia que en las noticias sobre violencias contra las mujeres $(82,82 \%$ y $72,56 \%$, respectivamente). En efecto, el autor presenta una imagen positiva en el $5,42 \%$ de las noticias sobre violencias contra las mujeres, frente al 1,03\% del resto de delitos. Por ejemplo, el pago de la fianza para conseguir la libertad provisional a la espera de juicio de un acusado de cuatro delitos (trata de seres humanos con fines de explotación sexual, difusión de pornografía infantil, abuso sexual de menores y delito contra la hacienda pública) fue presentado, en el 'lead' de la noticia, con unas declaraciones suyas, que realmente no le exculpan de los delitos atribuidos:

“«El director de porno Torbe sale de prisión bajo fianza»: «ìPor qué voy yo a violar a alguien si tengo a todas las tías que quiero?». Ésta es la frase que más ha repetido Ignacio Allende Fernández durante los 193 días que ha estado en prisión provisional. Ayer, el actor y productor porno conocido como Torbe quedó en libertad provisional” (El País, 06/11/2016).

A su vez, son también las informaciones sobre violencias machistas las que con mayor frecuencia (22,02\% de las noticias) muestran una imagen negativa del autor frente al 16,15\% del resto de delitos.

“«Torturada por negarse a ejercer la prostitución»: Será difícil que asimile cómo su propia familia no tuvo reparo en venderla y cómo su familia política la quiso convertir en una mercancía con la que hacer dinero" (El Mundo, 02/01/2012).

La valoración negativa del agresor en noticias sobre violencia de género comenzó tras la aprobación de las leyes que la combaten. De esa manera, la legislación incidió en el hecho de que las violencias contra las mujeres resultan ser las noticias en las que con menor frecuencia se presenta de manera neutra al agresor. Casi un cuarto de las noticias valora negativamente al autor, aunque destacan también por ser las informaciones en las que éste obtiene valoraciones positivas con mayor frecuencia.

Sin embargo, los medios optan por la presentación neutral de la víctima en todos los delitos. La imagen positiva de la misma únicamente destaca en los delitos contra la propiedad $(10,48 \%)$, terrorismo $(7,01 \%)$ y, en menor medida, violencias contra las mujeres $(4,96 \%)$. Además, la imagen negativa de las víctimas machistas, excluyendo agresiones sexuales, aumentó, por primera vez, a partir de 2008:

“«La Ertzaintza detiene en 14 horas a cinco hombres por violencia sexista»: Sobre las 13:00, la Ertzaintza efectuó el segundo arresto, después de que un efectivo de la policía autonómica detectara que un hombre, de 46 años, sobre el que pesaba una orden de alejamiento, se encontraba en compañía de su mujer en la plaza Ander Deuna, en Sukarrieta (El País, 21/02/2012). 
“"Detienen a una 'sin papeles’ cuando denunciaba a su pareja en comisaría»: Agentes de la Policía Local de Pontevedra han detenido a una mujer colombiana por estar en situación irregular en España, cuando intentaba denunciar a su compañero sentimental por agresión psicológica" (El Mundo, 26/02/2008).

Así, cuando las valoraciones negativas del agresor se multiplicaron a partir de 2008, la aprobación de la legislación en materia de igualdad supuso a su vez un aumento de las valoraciones negativas de las propias víctimas.

En efecto, si atendemos a la atribución de responsabilidad de los medios, las noticias sobre delitos contra la propiedad destacan por subrayar en el $24,29 \%$ de los casos los antecedentes del agresor, frente al 4,52\% de media en las noticias sobre delitos en general. Son las noticias sobre violencia de género las que en segundo lugar aluden a los antecedentes del agresor (8,94\%, el doble de la media de todas las noticias sobre delitos). Así mismo, son las únicas que explicitan la adopción de medidas de protección o falta de las mismas $(17,88 \%)$. Sin embargo, las noticias de violencia de género destacan por aludir a la existencia de denuncia por parte de la víctima, siete veces más que el resto de informaciones (33,65\% y 4,74\%, respectivamente). De ese modo, en las noticias sobre violencia de género en el ámbito de la pareja o expareja resulta ser el único delito donde la atribución de la responsabilidad por parte del medio se dirige en mayor medida a la víctima que al Estado (alusión a medidas de protección y antecedentes del agresor). A partir de 2008, ambos periódicos triplican las noticias que incluyen los antecedentes del agresor y duplican la cuestión sobre la denuncia previa, pero sólo en El País aumenta la mención a las medidas de protección.

De la misma manera, las noticias de violencia de género y las de violencia machista fuera de la pareja son las que incluyen con mayor frecuencia alguna justificación de lo ocurrido: $38,47 \%$ y $18,38 \%$, frente al $4,31 \%$ del resto de delitos. Se debe tener en cuenta que ninguna de las noticias sobre terrorismo incluye justificación alguna. A partir de 2008, además, se incrementa el número de noticias sobre violencia de género que incluyen datos que tienden a justificar lo sucedido, por ejemplo:

“«Detenido un hombre que degolló a su compañera sentimental en Torrevieja»: El agresor llegó a casa borracho, según él mismo declaró. Instantes después, la pareja entabló una discusión. El hombre puso fin a la disputa asestando una cuchillada en la carótida de la mujer" (El País, 07/01/2008).

Si bien ambos diarios señalan en un número parecido de noticias la alusión a la denuncia previa o no de la víctima, el gráfico número 1 muestra las diferencias de atribución de responsabilidad a partir de la aprobación de las leyes de igualdad. El País señala tres veces con mayor frecuencia las medidas de protección correspondientes al Estado y casi el doble de veces, los antecedentes del agresor. El Mundo destaca por incluir alguna justificación a la violencia de género en más de la mitad de las noticias sobre la misma, especialmente la cuestión de una discusión previa a la agresión. 
Gráfico 1. Atribución de responsabilidad en noticias sobre violencia de género a partir de 2008

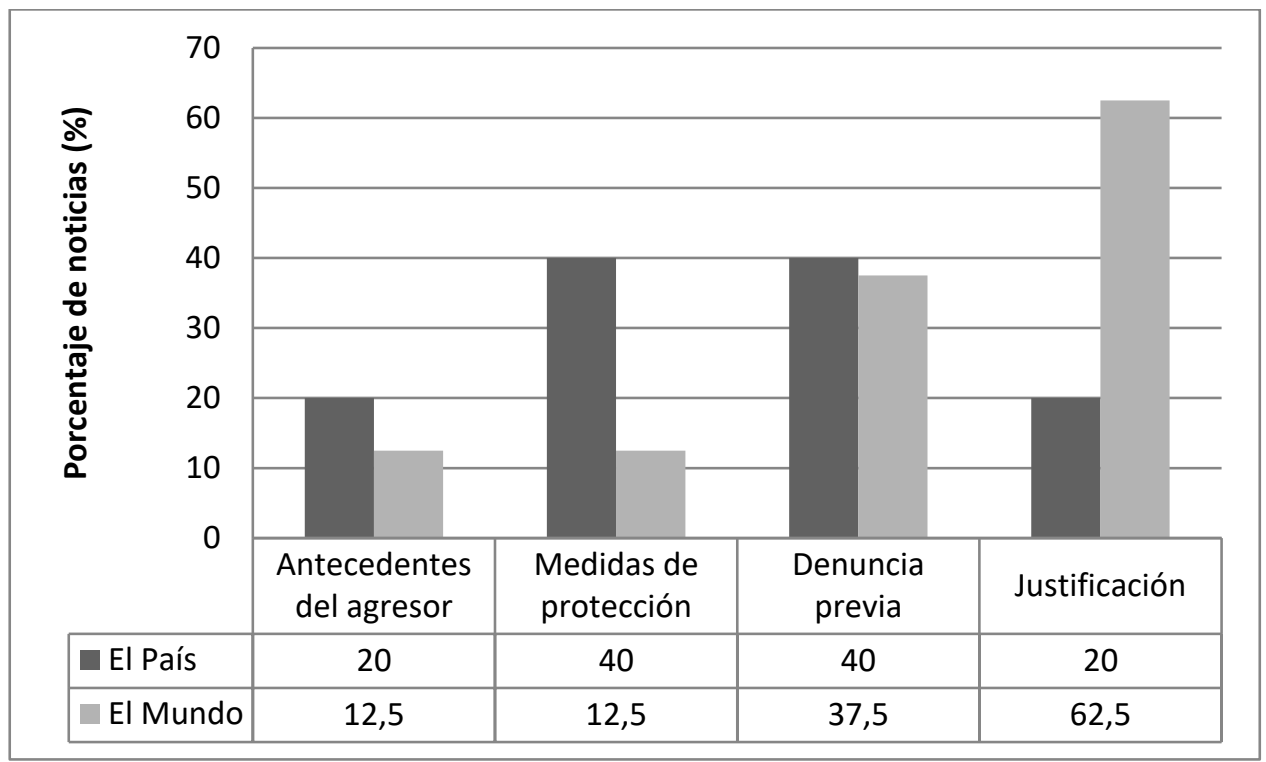

Fuente: elaboración propia

Sumando los datos de ambos diarios, las violencias contra las mujeres son las informaciones que más justificaciones del delito incluyen. Las noticias sobre violencia de género aportan algún tipo de justificación hasta diez veces con mayor frecuencia que el resto de noticias y la violencia machista fuera de la pareja, más de cuatro veces.

Finalmente, si analizamos los hechos relatados en las noticias sobre delitos, en cada noticia sobre violencias contra las mujeres aparecen las expresiones 'presunto', 'supuesto', 'presuntamente' y 'supuestamente' un total de 8,41 veces: 11,57, en violencia de género; 8,61, en violencia contra las mujeres fuera de la pareja; y 5,06 en agresiones sexuales. En el resto de noticias sobre delitos, tales expresiones se reducen a la mitad: 4,92 veces por noticia. Además, sólo el 9,45\% de estas expresiones utilizadas en noticias sobre violencias machistas es correcta y el 5,52\%, en el resto de delitos. En consecuencia, el empleo incorrecto de tales expresiones mantiene la tendencia de destacar por ser casi el doble en los delitos de violencias machistas: 7,62 veces de media en cada noticia sobre violencias contra las mujeres y 4,65, en el resto de informaciones sobre delitos cuyas víctimas no son únicamente mujeres:

“«La Ertzaintza desmonta con pruebas la versión del acusado de matar a su exnovia»: Fue entonces cuando [los agentes] descubrieron el cuerpo de C.E.B. tendido en el suelo, junto a un charco de sangre, ya sin vida. Tenía un cuchillo de 20 centímetros de hoja clavado en la parte posterior de la cabeza. El supuesto [sic] autor de la muerte había huido" (El País, 2012/5/31). 
Esta tendencia a emplear las expresiones 'presunto', 'supuesto', 'presuntamente’ y 'supuestamente' más frecuentemente en noticias sobre violencias contra las mujeres se incrementa tras la aprobación de las leyes de violencia de género: quince veces aumenta el empleo de tales expresiones en noticias sobre violencia machista fuera de la pareja y en agresiones sexuales, mientras que en las de violencia de género se eleva hasta veinte veces más a partir de 2008 , si bien en el resto de delitos esta práctica también aumenta de manera generalizada diez veces, especialmente en los delitos contra la propiedad. De nuevo, las noticias sobre la violencia de género que combate la Ley 1/2004 muestran el doble de 'presuntos' que el resto de noticias, seguidas de la violencia machista fuera de la pareja y agresiones sexuales. Incluso si atendemos meramente a la imputación del delito, los dos medios analizados utilizan las expresiones 'presunto delito' o 'supuesto delito' 7,43 veces por noticia en las informaciones de violencias contra las mujeres (10,43, en violencia de género) y únicamente 4,62 , en el resto de delitos.

De igual manera, los medios emplean diferentes expresiones para referirse al autor. Las más empleadas, agrupadas por tipo de delitos, nos muestran que el autor es identificado según el calificativo del delito, principalmente en las noticias sobre terrorismo ('terrorista': 1,77 veces por noticia, frente al 0,71 en el resto). El autor es nombrado según su situación procesal ('detenido', 'imputado'/'investigado') en mayor medida en las de violencia contra las personas sin componente de género (1,89 veces, frente a 1,09 en el resto). Finalmente, al autor se le presenta como 'presunto' seguido del calificativo del delito ('agresor', 'maltratador', 'violador') el doble de veces en las violencias contra las mujeres (0,56 veces por noticia y 0,21 , en el resto).

Lo mismo sucede con la denunciante, que aparece presentada como 'víctima' el doble de veces en noticias sobre violencias contra las mujeres que en el resto de delitos: 0,95 veces por noticia ( 1,7 veces, en violencia de género) y 0,47 por noticia sobre los otros delitos con víctimas. Ni siquiera las informaciones sobre terrorismo incluyen tantas veces la expresión 'víctima': únicamente 0,3 veces por noticia. De nuevo, al igual que en lo referente al empleo del término 'presunto', las noticias sobre violencias machistas suponen el doble que el resto también en la calificación de 'víctima' a la denunciante.

\section{Discusión}

Las noticias sobre violencias machistas han sido siempre muy numerosas, en relación al resto de delitos. Sólo fueron superadas por las informaciones sobre terrorismo. Sin embargo, Marín et al. subrayan que la cantidad de noticias sobre violencia de género en los medios no coincide con un "verdadero interés por sensibilizar a la sociedad" (2011: 436). En efecto, un estudio estadounidense de 2000 comprobó que las noticias sobre violencia sin componente de género eran más numerosas y más largas que las informaciones sobre violencia de género (McManus y Dorfman, 2005: 51). En El País, El Mundo y periódicos regionales de Cataluña, de 2006 a 2014, éstas seguían siendo noticias breves: "el 67\% no superan una columna de extensión" (Carrasco, Corcoy y Puig, 2015: 88). En esta investigación hemos comprobado, además, que la iniciativa informativa es desde la aprobación de las leyes de igualdad, en gran medida, ajena a la redacción y hay menos mujeres periodistas tratando estos temas a partir de 2008 o, por lo menos, firmando esas informaciones. Por ello, independientemente del número de noticias, el tratamiento informativo juega un papel fundamental en la consideración social de las violencias contra las mujeres. 
Diversos estudios han señalado que El País y El Mundo ofrecen más datos del autor que de la víctima en las noticias sobre violencia de género (Carrasco et al., 2015: 88; Menéndez, 2014: 69). En esta investigación se ha cuantificado que los medios ofrecen el doble de datos del autor de violencias contra las mujeres que del resto de delitos. Igualmente, el agresor en violencias machistas aparece incluso en el titular de la noticia también el doble de veces que en el resto de delitos. De esa manera, el origen del autor aparece como primer dato identificativo hasta cuatro veces más en violencias machistas, especialmente en agresiones sexuales, donde se le identifica según su lugar de procedencia más de diez veces que en el resto de delitos. De hecho, la mención al origen del autor es más frecuente en violencias contra las mujeres, mientras que el origen de la víctima lo es en el resto de delitos y no, en las informaciones sobre violencias machistas, si bien la procedencia de agresor y víctima aparece en la propia información de agencia cuando son extranjeros (Rodríguez Cárcela y López Vivas, 2020: 49). Teniendo en cuenta que otras investigaciones han explicado que El País destaca por no señalar "la etnia o nacionalidad de los implicados" (Marín et al., 2011: 462), debemos considerar que la actividad periodística de los dos periódicos de referencia analizados en la presente investigación puede ser la más respetuosa en el tratamiento de las violencias contra las mujeres.

Por su parte, las víctimas de violencia de género aparecen identificadas con nombre y apellidos, en base a la relación con el agresor, en mayor número de noticias que el detenido, especialmente a partir de 2008. También se incluyen más datos de ellas que de los agresores, sobre todo en noticias de violencia de género y violencia machista fuera de la pareja, a pesar de que esos agresores son los que más datos muestran.

Consiguientemente, atendiendo al primer encuadre analizado, comprobamos que las noticias sobre violencias machistas identifican a la víctima con mayor frecuencia que el resto de delitos. La identificación de la víctima resulta característica en las noticias sobre violencias machistas, especialmente, en violencia de género y, de manera considerable, desde la aprobación de la ley que pretende combatirla. También los medios aplican el segundo encuadre de manera característica en las violencias contra las mujeres, ya que los datos que ofrecen del agresor tienden a subrayar su alteridad racial con mayor incidencia que en el resto de noticias.

En ese sentido, los agresores y víctimas de violencias machistas son presentados de manera no neutral en mayor grado que en el resto de noticias sobre otros delitos. Mientras que la valoración negativa del agresor se evidencia a partir de 2008, esa tendencia se corresponde con la mayor valoración negativa también de la víctima. Resulta significativo que la persona cuya integridad pretende proteger la legislación en materia de igualdad sea la peor valorada en las noticias.

La valoración negativa de la víctima está relacionada con la atribución de responsabilidad de los medios. Así, los antecedentes del agresor de las violencias machistas son mencionados el doble que en el resto de delitos, sólo superadas por las noticias sobre los delitos contra la propiedad, que lo mencionan hasta tres veces más que en las violencias machistas. Pero, partir de 2008, se triplican las menciones a los antecedentes del agresor en las informaciones sobre violencias contra las mujeres. Así mismo, las cuestiones relativas únicamente a violencia de género, como la denuncia previa, dobla su presencia a partir de la legislación relativa a la misma. Sin embargo, la justificación es casi diez veces mayor en violencia de género que en el resto de delitos y cuatro veces más, en la violencia fuera de la pareja. 
La cuestión de los antecedentes del agresor mitiga el numeroso conjunto de noticias que incluyen la justificación de lo acontecido. Ambos indicadores muestran una clara tendencia a ser incluidos en las noticias sobre violencias contra las mujeres. Además, ambos se han incrementado tras la aprobación de las leyes contra la violencia de género. No obstante, el interrogante sobre la denuncia previa señala directamente a la víctima y, en efecto, esta última cuestión es más frecuente que las dos anteriores relativas al agresor. Igualmente, se potencia a partir de 2008. Aun así, entendemos que no es suficiente para considerar que el tercer encuadre sobre la atribución de la responsabilidad del delito a la víctima resulta específico en las noticias sobre violencias contra las mujeres

Varias autoras consideran que la presentación de la víctima como débil y carente de agencia implica que los medios le responsabilizan de la situación que experimenta (Gómez Nicolau, 2016: 197; Ménendez, 2014: 66). Ni tan siquiera considerando el hecho que a las mujeres se les denomina víctimas el doble de veces que a los denunciantes del resto de delitos compartimos que se trate de una atribución directa de responsabilidad a éstas.

Finalmente, el empleo del término 'presunto' en las noticias sobre violencias machistas dobla al resto de informaciones, siendo las noticias sobre violencia de género regulada por la ley de 2004 las que más 'presuntos' incluyen. A partir de 2008 se refleja un aumento desmesurado del empleo del término, que llega a ser diez veces más frecuente en el resto de delitos. De nuevo, en violencia de género se dobla esa cifra y en las noticias sobre violencia machista fuera de la pareja y agresiones sexuales, es 1,5 veces más frecuente. Lo mismo sucede con la denominación del autor, que incluye el término 'presunto' el doble de veces en las noticias sobre violencias machistas.

Por lo tanto, se ha demostrado que el cuarto encuadre sobre el menoscabo de la credibilidad de la denunciante resulta específico en las noticias sobre violencias contra las mujeres, al presentar una valoración negativa de la misma y al sembrar la duda sobre la veracidad de su testimonio, utilizando el doble de veces los términos 'presunto' y 'posible', tanto en el relato de lo denunciado, como en la identificación del agresor. Incluso la incorrecta expresión 'presunto delito' se da el doble de veces en violencias contra las mujeres que en el resto. Además, esta tendencia se ha recrudecido a partir de la aprobación de las leyes de igualdad.

Queda evidenciado que las noticias sobre violencias contra las mujeres adolecen de encuadres específicos (identificación más detallada de la denunciante que del agresor, construcción de la alteridad racial del agresor y menoscabo de la credibilidad de la denunciante). Así mismo, los medios mantienen una posición ambivalente respecto a la atribución de responsabilidad del delito a la denunciante $\mathrm{o}$ al agresor.

Es más, tras la aprobación de la ley de violencia de género, se desencadena una reacción mediática hacia las denunciantes de violencias machistas, más virulenta aún, en casos de violencia de género contemplada en la propia ley. Los medios ofrecen una peor valoración de estas víctimas, al mismo tiempo que acrecientan las características informativas que merman su credibilidad. La desacreditación del testimonio de la denunciante actúa como resistencia machista ante el avance de la legislación en materia de igualdad y tiene un efecto directo en la valoración social de las violencias contra las mujeres. Se trata del relato que guiará a la sociedad en la reflexión sobre el derecho a una vida libre de violencias, también para las mujeres. 


\section{Referencias bibliográficas}

Ardèvol-Abreu, A. (2015). Framing o teoría del encuadre en comunicación. Orígenes, desarrollo y panorama actual en España. Revista Latina de Comunicación Social, 70, 423-450. Disponible en http://doi.org/10.4185/RLCS-2015-1053

Berganza, M.R. (2003). La construcción mediática de la violencia contra las mujeres desde la Teoría del Enfoque. Comunicación y Sociedad, 16 (2), 9-32. Disponible en https://doi.org/10.15581/003.16.2.9-32

Carbadillo, P.C. (2010). El proceso de construcción de la violencia contra las mujeres: Medios de comunicación y movimiento feminista. Una aproximación desde la teoría del framing. (Tesis doctoral). Universitat Jaume I, España. Disponible en https://bit.ly/3bSvqEQ

Carrasco Rocamora, M., Corcoy Rius, M., Puig Mollet, M. (2015). El tratamiento de la violencia machista en la prensa de información general catalana. Estudio de dos casos mediáticos y su repercusión en la prensa local. Revista Internacional de Comunicación y Desarrollo, 2, 77-92. Disponible en http://dx.doi.org/10.15304/ricd.1.2.247

Consejo General del Poder Judicial (CGPJ) (2017). Memoria anual (correspondiente al ejercicio 2016). Disponible en https://bit.ly/3cyEBt4

De Frutos, R.A. (2015). Mediciones e índices de evaluación inclusivos: Examen de los indicadores de género y medios de comunicación. Cuadernos artesanos de comunicación, 86, 101-130. Disponible en http://doi.org/10.4185/cac86

De Noronha, L. (2018). The Figure of the 'Foreign Criminal': Race, Gender and the FNP. En M. Bhatia et al. (eds.), Media, Crime and Racism (pp. 337-354), Palgrave Studies in Crime, Media and Culture. Disponible en https://doi. org/10.1007/978-3-319-71776-0_17

Easteal, P., Holland, K., y Judd, K. (2015). Enduring themes and silences in media portrayals of violence against women. Women's Studies International Forum, 48, 103-113. Disponible en http://dx.doi.org/10.1016/j.wsif.2014.10.015

Escribano, M.I. (2014). Encuadres de la violencia de género en la prensa escrita y digital, nacional y regional. La Verdad, La Opinión, El Mundo y El País desde la teoría del framing (2005-2010). Murcia: Universidad de Murcia. Tesis doctoral. Disponible en https://bit.ly/3eKcfPb

El Mundo (2002). Libro de Estilo. Disponible en https://bit.ly/3vyNkE8

El País (2014). Libro de Estilo, Santillana: Madrid.

Fundéu BBVA (29 de julio de 2016). 'Presunto' es sinónimo de 'supuesto', Recomendaciones. Disponible en http://www. fundeu.es

Fundéu BBVA (13 de octubre de 2011). 'Al parecer' o 'presunto', pero no 'al parecer presunto', Recomendaciones. Disponible en http://www.fundeu.es

Giménez, P. y Berganza, M.R. (2009). Género y medios de comunicación. Un análisis desde la objetividad y la teoría del 'framing'. Madrid: Editorial Fragua.

Gómez Nicolau, E. (2016). Culpabilización de las víctimas y reconocimiento: límites del discurso mediático sobre la violencia de género. Feminismo/s, 27, pp. 197-218. Disponible en https://doi.org/10.14198/fem.2016.27.11

92 | nº 32, pp. 75-94 | doxa.comunicación

enero-junio de 2021 
Gor, F. (22 de marzo de 1998). ¿Presunto? Mejor, supuesto. El País. Disponible en https://bit.ly/2NoyxLf

Gorosarri, M. (24 de mayo de 2016). "Presuntitis": Los medios y las denuncias falsas. +Pikara y Eldiario.es. Disponible en: https://bit.ly/3eCEIF4

Judd, K., Easteal, P. (2013). Media Reportage Of Sexual Harassment: The (In)Credible Complainant. The Denning Law Journal, 25, pp. 1-17.

Krippendorff, K. (2018): Content Analysis: An Introduction to Its Methodology. Los Angeles: Sage.

Lacy, S., Watson, B. R., Riffe, D. y Lovejoy, J. (2015). Issues and Best Practices in Content Analysis. Journalism \& Mass Communication Quarterly, 92(4), 791-811. Disponible en https://doi.org/10.1177/1077699015607338

Lacy, S., Riffe, D., Stoddard, S., Martin, H.y Chang, K.K. (2001). Sample Size For Newspaper Content Analysis in Multi-Year Studies. Journalism \& Mass Communication Quaterly, 78(4), 836-845. Disponible en http://dx.doi. org/10.1177/107769900107800414

Ley Orgánica 1/2004, de 28 de diciembre, de Medidas de Protección Integral contra la Violencia de Género. Boletín Oficial del Estado, núm. 313, de 29 de diciembre de 2004, 42166-42197. Disponible en https://bit.ly/3vtn7qB

Ley Orgánica 3/2007, de 22 de marzo, Para la Igualdad Efectiva de Mujeres y Hombres. Boletín Oficial del Estado, núm. 71, de 23 de marzo de 2007, 12611-12645. Disponible en https://bit.ly/3vsSlhE

Luke, D., Caburnay, C. y Cohen, E. (2011). How Much Is Enough? New Recommendations for Using Constructed Week Sampling in Newspaper Content Analysis of Health Stories. Communication Methods and Measures, 5(1), 76-91. Disponible en http://dx.doi.org/10.1080/19312458.2010.547823

Marín, F., Armentia, J.I., y Caminos, J. (2011). El tratamiento informativo de las víctimas de violencia de género en Euskadi: Deia, El Correo, El País y Gara (2002-2009). Comunicación y Sociedad, 24(2), pp. 435-466.

McCombs, M. (2006). Estableciendo la agenda: El impacto de los medios en la opinión pública y en el conocimiento. Madrid: Grupo Planeta.

McManus, J., Dorfman, L. (2005). Functional Truth Or Sexist Distortion? Assessing A Feminist Critique Of Intimate Violence Reporting. Journalism, 6(1), pp. 43-65. Disponible en https://doi.org/10.1177/1464884905048952

Menéndez, M.I. (2014). Retos periodísticos ante la violencia de género. El caso de la prensa local en España. Comunicacion y Sociedad (Guadalajara), 22, pp. 52-77. Disponible en https://bit.ly/3vC7xsH

Nieva Fenoll, J. (2016). La razón de ser de la presunción de inocencia: el prejuicio social de culpabilidad. InDret, 1.

Peris Vidal, M. (2016). La representación rigurosa del origen de la violencia machista en la prensa escrita: una propuesta de medición. Estudios Sobre El Mensaje Periodístico, 22(2), 1123-1142. Disponible en https://doi.org/10.5209/ESMP.54255

Rodríguez Cárcela, R., López Vivas, A. (2020). Tratamiento informativo de la violencia de género: asesinatos de mujeres. Análisis de la agencia EFE. Ámbitos: Revista Internacional de Comunicación, 47, pp. 23-60. Disponible en http://dx.doi. org/10.12795/Ambitos.2020.i47.02

RTVE (2008). Manual de Estilo. Disponible en https://bit.ly/3vyZCN8 
Sánchez-Vera, J. (2012). Variaciones sobre la presunción de inocencia: Análisis funcional desde el derecho penal. Madrid: Marcial Pons.

Sutherland, G., McCormack, A., Easteal, P., Holland, K. y Pirkis, J. (2016). Media Guidelines For The Responsible Reporting Of Violence Against Women: A Review Of Evidence And Issues. Australian Journalism Review, 38(1), 5-17.

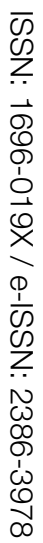

Tiscareño García, E. y Miranda Villanueva, O.M. (2020). Víctimas y victimarios de feminicidio en el lenguaje de la prensa escrita mexicana. Comunicar, 63(27), 51-60. Disponible en https://doi.org/10.3916/C63-2020-05

Zurbano Berenguer, B. (2018). Comunicación, periodismo y violencias contra las mujeres en España: Reflexiones en torno a un estado de la cuestión. Observatório, 4(2), 80-117. Disponible en http://dx.doi.org/10.20873/uft.2447-4266.2018v4n2p80 